\title{
Special Issue: Uncertainties in geometric design
}

In computing, it is a basic fact that, owing to the finiteness in representation, errors occur and they accumulate. In geometric computing, the 'logic' for performing set operations is not necessarily the classical binary one. How, then, does one ensure a robust process, if not error-free results? Physically, uncertainty in geometric data manifests itself in metrological studies in the small, and in robotic assembly in the large. How does one model the geometrical uncertainties so that the results of the computation can be applied to the real world? These two questions form the theme of this special issue of ComputerAided Design.

In this issue, seven papers are presented in three categories: fundamentals, methodologies and applications.

In the fundamentals category, 'Floating-point numbers with error estimates' by G Masotti examines error bounds, and proposes an architecture for a floating-point processor. The second paper, 'Robustness of numerical methods in geometric computation when problem data is uncertain' by $\mathbf{H}$ Desaulniers and N F Stewart shows the importance of the hypothesis in representation and the choice of metric.

In the methodology category, the paper 'Robust polygon modelling' by $\mathbf{V}$ Milenkovic provides a set of algorithms for performing set operations on polygonal regions, while the paper 'Robustness in solid modelling: a tolerance-based intuitionistic approach' by $\mathbf{S}$ Fang, B Bruderlin and $X$ Zhu identifies a set of geometric relations that are central to robustness, demonstrated via set operations on planar and natural quadric surfaces.

In the applications category, the paper 'Representational primitives for geometric tolerancing' by $\mathbf{J}$ Guilford and $\mathrm{J}$ Turner offers a condensed set of descriptors for tolerancing a geometric model, while the paper 'Solid model generation from measured point data' by $\mathbf{P}$ Chivate and A Jablokow attempts the converse: from data to model. Finally, the paper 'Optimal tolerance allotment using a genetic algorithm and truncated Monte Carlo simulation' by $J$ Lee and $G$ E Johnson examines the integration of a multivariate probability-density function for tolerance allotment.

The dedication of the authors notwithstanding, this collaboration of work is made possible by the vision of the editors of the journal: Karen Hemingway, Les Piegl and John Woodwark, the guidance and counsel of the numerous reviewers, and Pam Linderman, whose watchful eyes keep me from drifting too far from home during this sabbatical year.

Tony $C$ Woo

University of Michigan

$U S A$ 